\title{
Impossibility of Fast Stable Approximation of Analytic Functions from Equispaced Samples*
}

Rodrigo B. Platte ${ }^{\dagger}$ Lloyd N. Trefethen $\ddagger$ Arno B. J. Kuijlaars ${ }^{\S}$

\begin{abstract}
It is shown that no stable procedure for approximating functions from equally spaced samples can converge exponentially for analytic functions. To avoid instability, one must settle for root-exponential convergence. The proof combines a Bernstein inequality of 1912 with an estimate due to Coppersmith and Rivlin in 1992.
\end{abstract}

Key words. Runge phenomenon, Gibbs phenomenon, interpolation, radial basis functions, Lanczos iteration

AMS subject classifications. 41A05, 41A17, 65D05

DOI. $10.1137 / 090774707$

I. Introduction. It is well known that the $n$-point polynomial interpolants in equally spaced points to a function $f$ on $[-1,1]$ do not necessarily converge as $n \rightarrow \infty$, even if $f$ is analytic. Instead one may see wild oscillations near the endpoints, an effect known as the Runge phenomenon. Moreover, even for functions $f$ where convergence should take place in theory, the exponential ill-conditioning of the interpolation operator causes divergence in floating point arithmetic. If one considers trigonometric instead of polynomial interpolants, then unless $f$ is periodic, convergence fails again because of the implicit discontinuity at \pm 1 , this effect being known as the Gibbs phenomenon.

Polynomial interpolants in Chebyshev points, by contrast, converge geometrically and stably for analytic $f$. The same goes for trigonometric interpolants in equispaced points if $f$ is analytic and 2-periodic.

*Received by the editors October 22, 2009; accepted for publication (in revised form) May 7, 2010; published electronically May 5, 2011. The research of the first two authors was funded by UK EPSRC grant EP/E045847/1.

http://www.siam.org/journals/sirev/53-2/77470.html

${ }^{\dagger}$ School of Mathematical and Statistical Sciences, Arizona State University, Tempe, AZ 852871804 (platte@math.asu.edu,http://math.asu.edu/ platte/).

‡Oxford University Mathematical Institute, 24-29 St Giles’, Oxford OX1 3LB, UK (trefethen@ maths.ox.ac.uk, http://www.maths.ox.ac.uk/ ${ }^{2}$ trefethen/).

$\S$ Department of Mathematics, Katholieke Universiteit Leuven, Celestijnenlaan 200 B, B-3001 Leuven, Belgium (arno.kuijlaars@wis.kuleuven.be, http://wis.kuleuven.be/analyse/arno/). The research of this author was funded by FWO-Flanders project G.0427.09, K.U. Leuven research grant OT/08/33, the Belgian Interuniversity Attraction Pole P06/02, and grant MTM2008-06689-C02-01 of the Spanish Ministry of Science and Innovation. 
The question thus arises, is there some other procedure for approximation from equally spaced samples that might be geometrically convergent and numerically stable for nonperiodic analytic functions? For example, can we achieve geometric convergence by using least-squares fits, or conformal changes of variables, or variable-order splines, or radial basis functions (RBFs), or rational functions, or perhaps some kind of nonlinear adaptive strategy inspired by essentially nonoscillatory (ENO) schemes Padé extrapolants or Gegenbauer expansions for resolving the Gibbs phenomenon?

Dozens of such approximation methods have been proposed and found to be better than global polynomial interpolation. Nevertheless, we shall show that no stable method whatsoever, linear or nonlinear, can converge geometrically as $n \rightarrow \infty$. Specifically, for functions analytic in a fixed region containing $[-1,1]$, no matter how big, exponential convergence implies exponential ill-conditioning. More generally, convergence at a rate $C^{-n^{\tau}}$ for $\tau \in\left(\frac{1}{2}, 1\right]$ and $C>1$ implies ill-conditioning of magnitude $C^{n^{2 \tau-1}}$.

Let us review some facts about polynomial interpolation; for details on this material see approximation theory texts such as [12]. We begin with interpolation in Chebyshev points, which we define by $x_{j}=\cos (j \pi /(n-1)), 0 \leq j \leq n-1$. (Other variants based on roots or extrema of orthogonal polynomials have similar properties.) If $f$ is analytic on $[-1,1]$, then it is analytic and bounded in some Bernstein ellipse $E_{\rho}$ with parameter $\rho>1$, defined as the open region in the complex plane bounded by the ellipse with foci \pm 1 and semiminor and semimajor axis lengths summing to $\rho$. In this case the polynomial interpolants in Chebyshev points satisfy $\left\|f-f_{n}\right\|_{[-1,1]} \leq 4\|f\|_{E_{\rho}} \rho^{1-n} /(\rho-1)$, where $\|\cdot\|_{[-1,1]}$ is the supremum norm over $[-1,1]$ and $\|\cdot\|_{E_{\rho}}$ is the supremum norm over $E_{\rho}$. Moreover, the process is numerically stable, since the associated Lebesgue constants are of size $O(\log n)$ [18], and the interpolant can be evaluated stably in floating point arithmetic by Salzer's barycentric formula [39], as shown by Higham [28]. These properties are the basis of the Chebfun software system for "computing numerically with functions instead of numbers," where polynomials of degrees in the tens of thousands are routinely used for practical computation [45].

For equispaced points, by contrast, polynomial interpolation fails. Even for analytic $f$, the interpolants do not always converge, let alone geometrically, as shown by Runge [38]. Moreover, the interpolation process is exponentially ill-conditioned, with Lebesgue constants of size about $2^{n}$, as shown first by Turetskii [46] and later independently by Schönhage [40]. This ill-conditioning means that even if $f$ is entire, so that the interpolants converge in theory, they will diverge rapidly on a computer, at least for values of $x$ near the endpoints, because of exponential amplification of rounding errors.

The approximations we consider are formalized as follows. We assume that a family of $n$-point grids for each $n \geq 1$ has been fixed, such as equispaced or Chebyshev. An approximation procedure $\left\{\phi_{n}\right\}$ is a family of mappings $\phi_{n}: C([-1,1]) \rightarrow C([-1,1])$, $1 \leq n<\infty$, with the property that $\phi_{n}(f)$ depends only on the values $f$ takes on the $n$-grid. That is, if $f$ and $g$ take the same values on the $n$-grid, then $\phi_{n}(f)=\phi_{n}(g)$. The functions $\phi_{n}$ are otherwise entirely arbitrary and, in particular, they need not be linear.

For a definition of numerical stability we take the following standard notion, which reduces to the Lebesgue constant when $\phi_{n}$ is linear. (Properly speaking we are working with conditioning rather than stability, considering sensitivity of $\left\{\phi_{n}\right\}$ to perturbations rather than the effects of rounding errors in implementing it.) The 
condition number for $\phi_{n}$, a number in the range $[0, \infty]$, is

$$
\kappa_{n}=\sup _{f} \lim _{\varepsilon \rightarrow 0} \sup _{0<\|\delta f\|_{n \text {-grid }} \leq \varepsilon} \frac{\left\|\phi_{n}(f+\delta f)-\phi_{n}(f)\right\|_{[-1,1]}}{\|\delta f\|_{n \text {-grid }}},
$$

where $\|\cdot\|_{n \text {-grid }}$ is the supremum norm over the $n$-point grid. This amounts to a supremum over $f$ of a local Lipschitz constant for the mapping $\phi_{n}$ at $f$.

We shall show that in the case of equispaced grids, if $\left\{\phi_{n}\right\}$ converges geometrically for analytic functions, then the numbers $\left\{\kappa_{n}\right\}$ grow geometrically. Similarly, convergence at a subgeometric rate $C^{-n^{\tau}}$ with $\tau>1 / 2$ implies the growth of $\kappa_{n}$ at a rate $C^{n^{2 \tau-1}}$. Thus the Runge and Gibbs phenomena cannot be circumvented, even through the use of functions other than polynomials and approximation strategies other than interpolation.

2. Examples of Approximation Procedures. Dozens of methods have been proposed for approximating analytic functions from equispaced samples [7, 8]. In practical regimes they are sometimes very useful, a huge advance over polynomial interpolation. A number of their authors make claims of exponential convergence. But something must always go wrong as $n \rightarrow \infty$ : slow convergence, or ill-conditioning, or both. We briefly list some of these ideas without giving details.

1. Global polynomial interpolation. As discussed above, this is neither convergent nor stable [38, 40, 46].

2. Least-squares fitting by polynomials. Suppose the $n$ data values are used to determine a least-squares fit by a polynomial of some degree $d \ll n-1$. This approach weakens the Runge effect and hence makes the process more stable. However, one cannot achieve the goal this way. If $d=O(\sqrt{n})$, it may be possible to eliminate the numerical instability entirely, but then one has convergence at the rate $O\left(C^{-\sqrt{n}}\right)$ rather than $O\left(C^{-n}\right)$. If $d$ is bigger than this, one loses stability. See [36] for a quantification of this tradeoff and [9] for numerical experiments.

3. "Mock-Chebyshev" subsetting. Another approach is to interpolate by a polynomial of degree $d \ll n-1$ in some well-chosen subset of $d+1$ points [9, 29,42]. If the subset approximates a Chebyshev distribution, then one can achieve numerical stability in this fashion. However, again the convergence rate deteriorates to $O\left(C^{-\sqrt{n}}\right)$.

4. Regularization; high-degree polynomials. One of the major ideas in the field of data fitting is regularization, in which some of the available parameters are used not to fit the data per se, but to impose smoothness conditions. One variant of this idea explored by the first author (unpublished) is to fit data on the equispaced $n$-grid by a polynomial of degree $d \gg n-1$, using the extra $d+1-n$ coefficients to minimize a norm that measures the degree of oscillations. A similar method has been developed by Chandrasekaran et al. under the name of the Minimal Sobolev Norm scheme [10]. Perhaps the first contribution of this kind was by Boyd in 1992 [4].

5. Variable order splines. Splines of fixed orders converge for analytic functions, but only at an algebraic rate. To achieve faster convergence, one may consider splines of increasing orders. As with the previous ideas, there is necessarily a price to be paid in stability, particularly near the boundary.

6. Interpolation on subintervals. One can break $[-1,1]$ into subintervals and do polynomial interpolation on them. For any fixed $f$, there is a fixed subdivision which would have the effect of producing geometric convergence. However, this subdivision would depend on $f$, so it wouldn't be a procedure as defined above - and it would 
still be exponentially unstable. If the number of subintervals is allowed to grow with $n$ in such a way that each subinterval has order $n^{\beta}$ points for some $\beta \in(0,1)$, we get convergence of order $C^{-n^{\beta}}$ for every $C>1$ but at the cost of ill-conditioning of order $2^{n^{\beta}}$. Schemes of this kind are analyzed in [8].

7. Three-interval methods. One of the best strategies in practice may be to use one method on an interval $[-1+\epsilon, 1-\epsilon]$ for some $\epsilon>0$ coupled with a different approach in the intervals $[-1,-1+\epsilon]$ and $[1-\epsilon, 1]$. A method along these lines was proposed by Boyd [6], and a related scheme employing Chebyshev gridpoints in the end intervals was introduced by Platte and Gelb [35].

8. Conformal mappings and nonpolynomial interpolants. By a conformal change of variables as discussed by Hale [25] and Hale and Trefethen [26], one can construct interpolants that are better than polynomials on an equispaced grid. Indeed, by this method one can confine the Runge phenomenon to a neighborhood of \pm 1 as small as desired. It is impossible, however, to eliminate the bad neighborhood entirely.

9. Barycentric rational interpolants. Baltensperger, Berrut, and Noël showed that rational interpolants represented barycentrically can form highly accurate approximations [1], with geometric convergence on Chebyshev grids and their images under certain conformal maps. Floater and Hormann generalized these interpolants to higher orders on general grids [19]. However, if the grid is equispaced, then instabilities necessarily appear.

10. Adaptive rational functions. Work of Tee, Hale, and the second author builds upon the barycentric rational ideas just mentioned to produce highly nonlinear approximation schemes that first locate difficult regions of a function automatically by Padé or other techniques, then introduce a conformal map tuned to these regions, then approximate by barycentric rational interpolants associated with this conformal map $[44,25]$. This strategy proves very effective for dealing with localized regions of difficult behavior in $[-1,1]$.

11. Radial basis functions. A powerful generalization of polynomial interpolants is radial basis functions (RBFs), a major technology in scientific computing that offers high accuracy combined with geometric flexibility. Certain families of RBFs with a shape parameter $\varepsilon$ provide very high accuracy $[14,48]$. With equispaced data, however, invariably one finds that values of $\varepsilon$ which produce high accuracy also suffer from high ill-conditioning $[22,33,34]$. Various techniques can alleviate the problems [20, 21], but it follows from our theorem that they can never be eliminated entirely.

12. Adaptive algorithms related to ENO. In the field of numerical solution of conservation laws, a longstanding theme has been the introduction of flux limiters and other nonlinear strategies to suppress oscillations. ENO, which stands for essentially nonoscillatory, is an idea in this area due to Harten and others in which difference stencils are chosen adaptively to minimize oscillations [27]. Berzins has shown that some of these techniques are also useful for problems of function approximation and, in particular, may be effective at suppressing Runge effects [3].

13. Wang's rational regression scheme. Wang, Moin, and Iaccarino have proposed a scheme for approximation by high-order rational functions in which a different griddependent regression calculation is performed at each point where an approximant needs to be evaluated, making the approximations $\phi_{n}$ very complicated indeed but for some functions highly accurate. The scheme applies to arbitrary grids, including equally spaced ones, and a theorem is given on superpolynomial convergence [47].

14. Methods based on overcoming the Gibbs phenomenon. For periodic analytic functions, Fourier analysis (trigonometric interpolation) is a stable algorithm with 
geometric convergence. Thus, to get geometric convergence for an analytic function on $[-1,1]$, we need "only" find a way around the Gibbs phenomenon implicit in the discontinuity in $f$ between -1 and 1 . There is quite a literature on beating the Gibbs phenomenon. One approach is the Gegenbauer method of Gottlieb and coauthors [24], but Boyd has shown that either geometric convergence or stability must be sacrificed as $n \rightarrow \infty$ [5]. Related contributions are due to Gelb, Tadmor, and Tanner [23, 43]. A Padé algorithm was proposed in [13], and further contributions in this area include [30] and [41]. For an excellent survey of much of this subject see [43].

3. Theorems. We now state and prove the main theorem (Theorem 3.1) and a generalization (Theorem 3.4). These theorems involve analytic functions, and the first thing to do is set down a standard definition about families of such functions. If $E$ is a compact set in the complex plane, we define $B(E)$ to be the Banach space of functions continuous on $E$ and analytic in the interior, with norm $\|f\|_{E}=\sup _{z \in E}|f(z)|$.

Theorem 3.1 takes the following form: exponential convergence implies exponential instability. Here is the precise statement.

THEOREM 3.1. Let a compact set $E$ containing $[-1,1]$ in its interior be fixed, and suppose $\left\{\phi_{n}\right\}$ is an approximation procedure based on equispaced $n$-grids such that, for some $M<\infty$ and $\sigma>1$,

$$
\left\|f-\phi_{n}(f)\right\|_{[-1,1]} \leq M \sigma^{-n}\|f\|_{E} \quad(1 \leq n<\infty)
$$

for all $f \in B(E)$. Then the condition numbers for $\phi_{n}$ as defined in (1.1) satisfy

$$
\kappa_{n} \geq C^{n}
$$

for some $C>1$ and all sufficiently large $n$.

Although this theorem has nothing to do with polynomials, our proof is based on combining two lemmas that deal with polynomials. We present the lemmas first, and then give the proof of the theorem. The caption of Figure 3.1 gives a summary of the whole argument.

The first lemma is due to Bernstein in 1912 [2, sec. 9]. If $p$ is a polynomial of degree $d$, then it is not hard to show that the maximum of $|p(z)|$ on the disk of radius $R>1$ is no greater than $R^{d}$ times the maximum of $|p(z)|$ on the unit disk. Bernstein's lemma generalizes this result from the unit disk to the unit interval. We continue to let $E_{\rho}$ denote the Bernstein ellipse defined in section 1, with $\rho>1$.

LEMMA 3.2 (Bernstein). If $p$ is a polynomial of degree $d$, then

$$
\|p\|_{E_{\rho}} \leq \rho^{d}\|p\|_{[-1,1]} .
$$

Proof. The function $z \mapsto z+\left(z^{2}-1\right)^{1 / 2}$ is a conformal map of $\mathbb{C} \backslash[-1,1]$ onto the exterior of the unit disk. In particular, it is analytic in $\mathbb{C} \backslash[-1,1]$, with a simple pole at $z=\infty$. If $p$ is a polynomial of degree $d$, it follows that the function $q(z)=$ $p(z) /\left(z+\left(z^{2}-1\right)^{1 / 2}\right)^{d}$ is analytic throughout $\mathbb{C} \cup\{\infty\} \backslash[-1,1]$. By the maximum modulus principle, for any $z \in \mathbb{C} \cup\{\infty\} \backslash[-1,1]$ we accordingly have $|q(z)| \leq\|q\|_{[-1,1]}$.

Now, for any $\rho>1$, the set of complex numbers satisfying

$$
\left|z+\left(z^{2}-1\right)^{1 / 2}\right|=\rho
$$

is precisely the ellipse bounding $E_{\rho}$. Thus, the inequality just derived amounts to the statement that for any $z$ on the boundary of $E_{\rho}$,

$$
|p(z)| / \rho^{d} \leq\|p\|_{[-1,1]} .
$$

This establishes (3.3). 


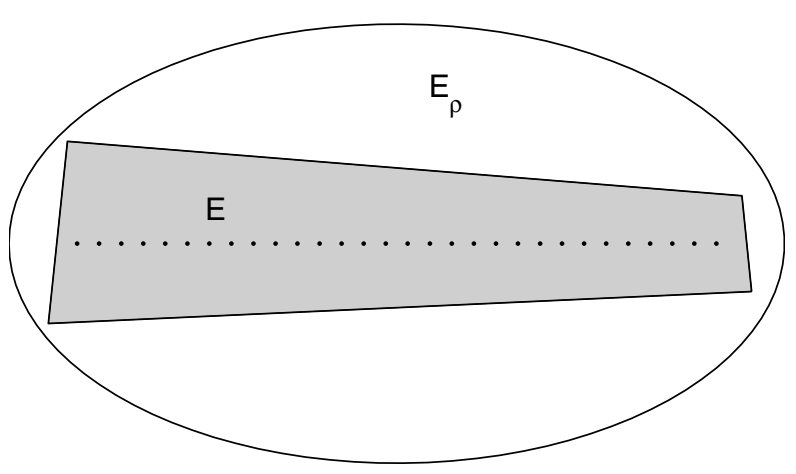

Fig. 3.I In outline, the proof of Theorem 3.1 goes like this. Suppose an approximation procedure $\left\{\phi_{n}\right\}$ gives errors $\left\|f-\phi_{n}(f)\right\|_{[-1,1]} \leq C^{-n}\|f\|_{E}$ for $f$ analytic on some set $E$ containing $[-1,1]$. Then it also gives errors $\leq C^{-n}\|f\|_{E_{\rho}}$ for $f$ analytic on a Bernstein $\rho$-ellipse $E_{\rho}$ enclosing $E$. In particular, the error is $\leq C^{-n}\|p\|_{E_{\rho}}$ for any polynomial $p$ of degree $d$. By Lemma 3.2, due to Bernstein, this error bound is in turn bounded by $C^{-n} \rho^{d}\|p\|_{[-1,1]}$. Fixing $d=\alpha n$ for sufficiently small $\alpha>0$ converts this to $C^{-n}\|p\|_{[-1,1]}$ with a new constant $C$. In particular, for large enough $n$, the approximation $\phi_{n}(p)$ has norm on $[-1,1]$ at least half that of $p$. But by Lemma 3.3, due to Coppersmith and Rivlin, some polynomials of degree $\alpha n$ are exponentially larger on $[-1,1]$ than on the equispaced $n$-grid as $n \rightarrow \infty$. It follows that for these polynomials, the size of $\phi_{n}(p)$ on $[-1,1]$ must be exponentially larger than the size of $p$ on the $n$-grid, and this implies that the condition numbers of the approximation procedure are exponentially large.

The second lemma deals with the question, how big can a polynomial $p$ of degree $d<n$ be on $[-1,1]$ if it is bounded in absolute value by 1 on the equispaced $n$ grid? If $d=n-1$, we know that $p$ can be huge, of order $2^{n}$; this is the case of the exponentially large Lebesgue constants of the Runge phenomenon, analyzed by Turetskii and Schönhage as mentioned earlier. But what if $d$ is, say, $n / 2$ or $n / 10$ ? The answer is that $p$ can still be very large so long as $d$ is larger than $O(\sqrt{n})$. The literature on this problem goes back at least to Schönhage, Ehlich, and Zeller in the early 1960s [15, 16, 17, 40]. Rather precise answers were later given by Coppersmith and Rivlin in a paper of 1992 [11], with further developments due to Rakhmanov [36]. In particular, the theorem of [11], translated to our setting, goes as follows.

Lemma 3.3 (Coppersmith and Rivlin). There exist universal constants $C_{1}>1$, $C_{2}>1$, and $n_{1} \geq 1$ such that

$$
C_{1}^{d^{2} / n} \leq \sup _{\operatorname{deg} p=d} \frac{\left\|p_{d}\right\|_{[-1,1]}}{\left\|p_{d}\right\|_{n-\text { grid }}} \leq C_{2}^{d^{2} / n}
$$

for all $n \geq n_{1}$ and $d \leq n-1$.

Proof. See [11].

We are now prepared to prove the main theorem.

Proof of Theorem 3.1. The set $E$ is contained in the Bernstein ellipse $E_{\rho}$ for sufficiently large $\rho$. If (3.1) holds for $B(E)$, it holds for $B(\tilde{E})$ whenever $E \subseteq \tilde{E}$, so without loss of generality let us now take $E$ to be $E_{\rho}$ for some $\rho>1$. For convenience we rewrite (3.1) in the equivalent form

$$
\left\|f-\phi_{n}(f)\right\|_{[-1,1]} \leq \frac{1}{2} \rho^{-\alpha n}\|f\|_{E} \quad\left(n_{0} \leq n<\infty\right)
$$

for some sufficiently large $n_{0}$ and some $\alpha>0$. 
Now for any integer $d \geq 0$, let $p_{d}$ be a polynomial of degree $d$. Since $p_{d}$ is analytic throughout the complex plane, it belongs to $B(E)$, and so (3.4) holds with $p_{d}$ in place of $f$. By Lemma 3.2 we have $\left\|p_{d}\right\|_{[-1,1]} \geq \rho^{-d}\left\|p_{d}\right\|_{E}$. If (3.4) holds, it then follows that

$$
\left\|\phi_{n}\left(p_{d}\right)\right\|_{[-1,1]} \geq\left\|p_{d}\right\|_{[-1,1]}-\left\|p_{d}-\phi_{n}\left(p_{d}\right)\right\|_{[-1,1]} \geq \frac{1}{2}\left\|p_{d}\right\|_{[-1,1]}
$$

whenever $\rho^{-\alpha n} \leq \rho^{-d}$ with $n \geq n_{0}$ or, equivalently, whenever $n \geq \max \left\{d / \alpha, n_{0}\right\}$. Combining this with the fact implied by (3.1) that $\phi_{n}(0)=0$, we get

$$
\left\|\phi_{n}\left(p_{d}\right)-\phi_{n}(0)\right\|_{[-1,1]} \geq \frac{1}{2}\left\|p_{d}\right\|_{[-1,1]} \quad \text { whenever } \quad n \geq \max \left\{d / \alpha, n_{0}\right\} .
$$

From the definition of the condition number, taking $f=0$ and $\delta f=\varepsilon p_{d}$ for any $\varepsilon>0$ in (1.1), this implies

$$
\kappa_{n} \geq \limsup _{\varepsilon \rightarrow 0} \frac{1}{2} \frac{\left\|\varepsilon p_{d}\right\|_{[-1,1]}}{\left\|\varepsilon p_{d}\right\|_{n \text {-grid }}}=\frac{1}{2} \frac{\left\|p_{d}\right\|_{[-1,1]}}{\left\|p_{d}\right\|_{n \text {-grid }}}
$$

for all $n \geq \max \left\{d / \alpha, n_{0}\right\}$. Another way to say it is this: for all $n \geq n_{0}$,

$$
\kappa_{n} \geq \frac{1}{2} \sup _{\operatorname{deg} p \leq \alpha n} \frac{\|p\|_{[-1,1]}}{\|p\|_{n \text {-grid }}} .
$$

The first inequality of Lemma 3.3 now implies that for some $C_{1}>1$,

$$
\sup _{\operatorname{deg} p \leq \alpha n} \frac{\left\|p_{d}\right\|_{[-1,1]}}{\left\|p_{d}\right\|_{n \text {-grid }}} \geq C_{1}^{\alpha^{2} n}
$$

for large enough $n$. Using this inequality in (3.5) we find that (3.2) holds with any $C>1$ such that $C<C_{1}^{\alpha^{2}}$, and this completes the proof.

Figure 3.2 illustrates the kind of Coppersmith-Rivlin polynomials we have just used in the proof: bounded on an equispaced grid, but much bigger between gridpoints.

It is interesting to consider the significance of (3.5) if $\alpha \geq 1$. In this case it would seem we could take $f$ to be a nonzero polynomial $p$ with zeros at all the gridpoints and conclude that $\kappa_{n}=\infty$ ! However, $\alpha \geq 1$ in (3.5) cannot occur. To see this we note that if $p$ were such a polynomial, then $\phi_{n}$ would give the same result for both $p$ and $-p$ since they take the same zero values on the grid. If we call this function $q$, we may infer that $|p(x)|+|q(x)| \leq \frac{1}{2} \rho^{-\alpha n}\|p\|_{E}$ for all $x \in[-1,1]$, hence $\|p\|_{[-1,1]} \leq \frac{1}{2} \rho^{-\alpha n}\|p\|_{E}$. But by Lemma 3.2 we also have $\|p\|_{[-1,1]} \geq \rho^{-n}\|p\|_{E}$, and combining these bounds gives $\rho^{-n} \leq \frac{1}{2} \rho^{-\alpha n}$, which is a contradiction if $\alpha \geq 1$.

The proof of Theorem 3.1 can be modified to establish a more general result. By replacing $\alpha n$ by $\alpha n^{\tau}$ throughout (we do not give details) one can show the following theorem.

TheOREM 3.4. Let the hypothesis of Theorem 3.1 be generalized to

$$
\left\|f-\phi_{n}(f)\right\|_{[-1,1]} \leq M \sigma^{-n^{\tau}}\|f\|_{E} \quad(1 \leq n<\infty)
$$

for some $\tau \in\left(\frac{1}{2}, 1\right]$. Then we have

$$
\kappa_{n} \geq C^{n^{2 \tau-1}}
$$



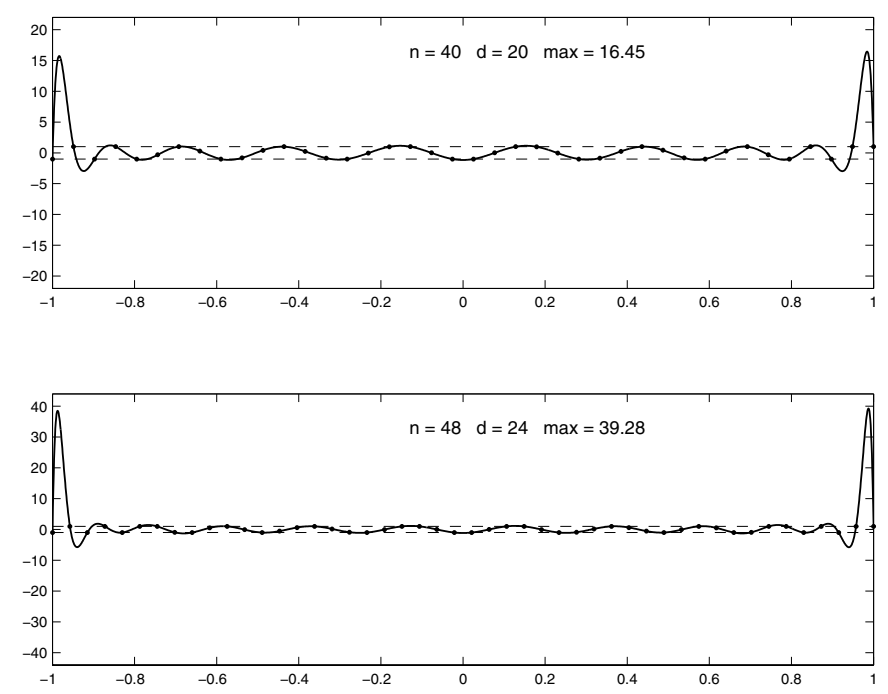

Fig. 3.2 Maximal polynomials $p$ of degree $n / 2$ on $[-1,1]$ for $n=40$ and 48 subject to the constraint $\left|p\left(x_{j}\right)\right| \leq 1$ on the equispaced $n$-grid. (One might have expected the optimal polynomials to be even, but, in fact, they deviate slightly from evenness.) As shown by Coppersmith and Rivlin [11], the maximum grows exponentially as $n \rightarrow \infty$, and this observation is a key step in the proof of Theorems 3.1 and 3.4.

4. Connections with Potential Theory and Matrix Iterations. The key point of the proof of Theorems 3.1 and 3.4 is that, for $\sqrt{n} \ll d \ll n-1$, there are polynomials of degree $d$ that are much larger on $[-1,1]$ than on the equispaced $n$-grid. Such polynomials can be constructed by taking the value to be 0 at a certain fraction of the outer points of the grid - with large oscillations between these points - while requiring the polynomial to be small in the inner regions of the interval. Their existence is related to the subject of potential theory in the complex plane [37]. Roughly speaking, polynomials can take exceptionally large values between gridpoints in regions of $[-1,1]$ where the gridpoints, if viewed as point charges, contain "too little charge" for an equilibrium distribution. Using potential theory, it is possible to work out an exact formula for the value of the ratio (3.5) in the limit $n \rightarrow \infty$ :

$$
\lim _{n \rightarrow \infty} \frac{1}{n} \log \sup _{\operatorname{deg} p \leq \alpha n} \frac{\|p\|_{[-1,1]}}{\|p\|_{n \text {-grid }}}=\frac{1}{2}[(1+\alpha) \log (1+\alpha)+(1-\alpha) \log (1-\alpha)]
$$

For example, if $\operatorname{deg} p=n / 2$, the ratios (3.5) are approximately $C^{n}$ with $C=$ $(27 / 16)^{1 / 4} \approx 1.140$. (A better approximation, with the same limiting values after taking logarithms, appears to be $C^{n} / n$.) We shall give details of this calculation elsewhere.

Curiously, the same potential theory connections, and the same polynomials, also arise in analysis of the convergence of the Lanczos iteration for computing eigenvalues of real symmetric matrices, and of other related iterations. Indeed, Figure 1 of [32] shows exactly the kind of polynomial at issue. As discussed in [32], the Lanczos iteration converges fast to eigenvalues in those regions of "too little charge." Thus we have connections like this: if an $n$-point grid in $[-1,1]$ corresponds to matrix eigenvalues, some of which the Lanczos iteration will find in $o(n)$ steps, then any rapidly converging function approximation procedure based on data from the same grid must have very large condition numbers. 
5. Discussion. We have kept this paper short in the interests of readability. Certain generalizations, however, suggest themselves. One of them, alluded to in the last paragraph, would be to consider nonequispaced grids. By arguments related to those here, one could presumably show that stability and geometric convergence are incompatible for arbitrary grids that fail to cluster enough points near \pm 1 . Proofs of such results would make use of the fact that the inequality (3.5) holds for arbitrary grids, not just equispaced.

Concerning the hypotheses of Theorems 3.1 and 3.4, a modification suggests itself. Might there be an approximation procedure that converges exponentially for each function $f$ analytic on $[-1,1]$, though without satisfying a uniform bound of the type (3.1)? For linear approximations it follows from our theorems that the answer is no, but we do not know if this conclusion carries over to the nonlinear case.

It is worth emphasizing that our analysis is based on measuring geometric convergence uniformly across the interval $[-1,1]$. Convergence on subintervals $[-1+\varepsilon, 1-\varepsilon]$ is another matter, for here the polynomials such as those of Figure 3.2 cannot be too large, as proved in [36]. In particular, though our theorems imply that the Gibbs phenomenon cannot be overcome uniformly at all points of the interval of approximation, pointwise success is not precluded.

Our results for approximation have analogues for quadrature formulas, which are based on approximations of various kinds. A stable quadrature formula is normally associated with positive weights, and it was shown by Wilson many years ago that if the grid is equispaced and the weights are positive, then the polynomial order of the formula can only scale as $\sqrt{n}[31,49]$.

A curious point arises concerning polynomial and nonpolynomial approximations. Traditionally, much of approximation theory has been based on polynomials in one way or another, and, in particular, the need to cluster interpolation points at the boundary is normally regarded as an effect associated with polynomials. Yet Theorems 3.1 and 3.4 are not about polynomials; they concern arbitrary approximations. It is perhaps puzzling then that the proof makes use of polynomials after all: a polynomial $p$ of degree $d$ is introduced with the crucial property that it is much smaller on the grid than on $[-1,1]$ (Lemma 3.3), yet not much bigger on the complex set $E$ than on $[-1,1]$ (Lemma 3.2). It seems clear that in principle it must not have been necessary to introduce polynomials in this proof; it is just that the available results for polynomials made it convenient to do so.

In closing we would like to reiterate that some of the methods mentioned in section 2 are highly effective in practice. Our theorems show that in a certain sense no method can achieve all one might dream of, but, like all theorems, they leave open the possibility that adjusted hypotheses might give adjusted conclusions.

Acknowledgments. We are grateful for advice from John Boyd, Bengt Fornberg, Daan Huybrechs, Colin Macdonald, Sheehan Olver, Endre Süli, Eitan Tadmor, Jared Tanner, and Joris Van Deun.

\section{REFERENCES}

[1] R. Baltensperger, J.-P. Berrut, And B. NoËL, Exponential convergence of a linear rational interpolant between transformed Chebyshev points, Math. Comp., 68 (1999), pp. 1109-1120.

[2] S. Bernstein, Sur l'Ordre de la Meilleure Approximation des Fonctions Continues par des Polynomes de Degré Donné, Mém. Acad. Roy. Belg., 1912.

[3] M. Berzins, Adaptive polynomial interpolation on evenly spaced meshes, SIAM Rev., 49 (2007), pp. $604-627$. 
[4] J. P. BoyD, Defeating the Runge phenomenon for equispaced polynomial interpolation via Tikhonov regularization, Appl. Math. Lett., 5 (1992), pp. 57-59.

[5] J. P. BOYD, Trouble with Gegenbauer reconstruction for defeating Gibbs' phenomenon: Runge phenomenon in the diagonal limit of Gegenbauer polynomial approximations, J. Comput. Phys., 204 (2005), pp. 253-264.

[6] J. P. BOYD, Exponentially accurate Runge-free approximation of non-periodic functions from samples on an evenly spaced grid, Appl. Math. Lett., 20 (2007), pp. 971-975.

[7] J. P. BOyd AND J. R. ONG, Exponentially-convergent strategies for defeating the Runge phenomenon for the approximation of non-periodic functions. Part I: Single-interval schemes, Comm. Comput. Phys., 5 (2009), pp. 484-497.

[8] J. P. Boyd And J. R. ONG, Exponentially-convergent strategies for defeating the Runge phenomenon for the approximation of non-periodic functions, Part II: Multi-interval Polynomial Schemes and multidomain Chebyshev interpolation, Appl. Numer. Math., 61 (2011), pp. $460-472$.

[9] J. P. Boyd AND F. XU, Divergence (Runge phenomenon) for least-squares polynomial approximation on an equispaced grid and Mock-Chebyshev subset interpolation, Appl. Math. Comput., 210 (2007), pp. 158-168.

[10] S. Chandrasekaran, K. R. Jayaraman, J. Moffitt, H. N. Mhaskar, and S. Pauli, Minimum Sobolev Norm schemes and applications in image processing, Proc. SPIE, 7535 (2010).

[11] D. Coppersmith And T. J. Rivlin, The growth of polynomials bounded at equally spaced points, SIAM J. Math. Anal., 23 (1992), pp. 970-983.

[12] P. J. DAVIS, Interpolation and Approximation, Dover, New York, 1975.

[13] T. A. Driscoll and B. Fornberg, A Padé-based algorithm for overcoming the Gibbs phenomenon, Numer. Algorithms, 26 (2001), pp. 77-92.

[14] T. A. Driscoll and B. Fornberg, Interpolation in the limit of increasingly flat radial basis functions, Comput. Math. Appl., 43 (2002), pp. 413-422.

[15] H. Ehlich, Polynome zwischen Gitterpunkten, Math. Z., 93 (1966), pp. 144-153.

[16] H. Ehlich ANd K. Zeller, Schwankung von Polynomen zwischen Gitterpunkten, Math. Z., 86 (1964), pp. 41-44.

[17] H. Ehlich ANd K. Zeller, Numerische Abschätzung von Polynomen, Z. Angew. Math. Mech., 45 (1965), pp. T20-T22.

[18] H. Ehlich AND K. Zeller, Auswertung der Normen von Interpolationsoperatoren, Math. Ann., 164 (1966), pp. 105-112.

[19] M. S. FloAter AND K. Hormann, Barycentric rational interpolation with no poles and high rates of approximation, Numer. Math., 107 (2007), pp. 315-331.

[20] B. Fornberg And C. Piret, A stable algorithm for flat radial basis functions on a sphere, SIAM J. Sci. Comput., 30 (2007), pp. 60-80.

[21] B. Fornberg AND G. Wright, Stable computation of multiquadric interpolants for all values of the shape parameter, Comput. Math. Appl., 48 (2004), pp. 853-867.

[22] B. Fornberg And J. Zuev, The Runge phenomenon and spatially variable shape parameters in RBF interpolation, Comput. Math. Appl., 54 (2007), pp. 379-398.

[23] A. Gelb and J. Tanner, Robust reprojection methods for the resolution of the Gibbs phenomenon, Appl. Comput. Harmon. Anal., 20 (2006), pp. 3-25.

[24] D. Gottlieb And C.-W. Shu, On the Gibbs phenomenon and its resolution, SIAM Rev., 39 (1997), pp. 644-668.

[25] N. Hale, On the Use of Conformal Maps to Speed up Numerical Computations, D.Phil. thesis, Oxford University Computing Lab., Oxford, 2009.

[26] N. Hale and L. N. Trefethen, New quadrature formulas from conformal maps, SIAM J. Numer. Anal., 46 (2008), pp. 930-948.

[27] A. Harten, B. Engquist, S. Osher, and S. R. Chakravarthy, Uniformly high order accurate essentially non-oscillatory schemes. III, J. Comput. Phys., 71 (1987), pp. 231-303.

[28] N. J. Higham, The numerical stability of barycentric Lagrange interpolation, IMA J. Numer. Anal., 24 (2004), pp. 547-556.

[29] P. Hoffman and K. C. Reddy, Numerical differentiation by high order interpolation, SIAM J. Sci. Stat. Comput., 8 (1987), pp. 979-987.

[30] D. HuYbrechs, On the Fourier extension of nonperiodic functions, SIAM J. Numer. Anal., 47 (2010), pp. 4326-4355.

[31] D. Huybrechs, Stable high-order quadrature rules with equidistant points, J. Comput. Appl. Math., 231 (2009), pp. 933-947.

[32] A. B. J. KulJlaArs, Convergence analysis of Krylov subspace iterations with methods from potential theory, SIAM Rev., 48 (2006), pp. 3-40.

Copyright $@$ by SIAM. Unauthorized reproduction of this article is prohibited. 
[33] R. B. Platte, How fast do radial basis function interpolants of analytic functions converge?, IMA J. Numer. Anal., to appear.

[34] R. B. Platte and T. A. Driscoll, Polynomials and potential theory for Gaussian radial basis function interpolation, SIAM J. Numer. Anal., 43 (2005), pp. 750-766.

[35] R. B. Platte and A. Gelb, A hybrid Fourier-Chebyshev method for partial differential equations, J. Sci. Comput., 39 (2009), pp. 244-264.

[36] E. A. Rakhmanov, Bounds for polynomials with a unit discrete norm, Ann. of Math. (2), 165 (2007), pp. 55-88.

[37] T. Ransford, Potential Theory in the Complex Plane, Cambridge University Press, Cambridge, UK, 1995.

[38] C. Runge, Über empirische Funktionen and die Interpolation zwischen äquidistanten Ordinaten, Z. Math. Phys., 46 (1901), pp. 224-243.

[39] H. E. SALzER, Lagrangian interpolation at the Chebyshev points $x_{n, \nu}=\cos (\nu \pi / n), \nu=0(1) n$; some unnoted advantages, Computer J., 15 (1972), pp. 156-159.

[40] A. Schönhage, Fehlerfortpflanzung bei Interpolation, Numer. Math., 3 (1961), pp. 62-71.

[41] B. D. Shizgal And J. H. Jung, Towards the resolution of the Gibbs phenomenon, J. Comput. Appl. Math., 161 (2003), pp. 41-65.

[42] G. Stengle, Chebyshev interpolation with approximate nodes of unrestricted multiplicity, J. Approx. Theory, 57 (1989), pp. 1-13.

[43] E. TADMOR, Filters, mollifiers and the computation of the Gibbs phenomenon, Acta Numer., 16 (2007), pp. 305-378.

[44] T. W. Tee And L. N. Trefethen, A rational spectral collocation method with adaptively transformed Chebyshev grid points, SIAM J. Sci. Comput., 28 (2006), pp. 1798-1811.

[45] L. N. Trefethen, N. Hale, R. B. Platte, T. A. Driscoll, and R. Pachón, Chebfun Version 3, http://www.maths.ox.ac.uk/chebfun/, Oxford University, Oxford, 2009.

[46] A. H. Turetskil, The bounding of polynomials prescribed at equally distributed points, Proc. Pedag. Inst. Vitebsk, 3 (1940), pp. 117-127 (in Russian).

[47] Q. WANG, P. MoIn, AND G. IACCARINO, A rational interpolation scheme with superpolynomial rate of convergence, SIAM J. Numer. Anal., 47 (2010), pp. 4073-4097.

[48] H. Wendland, Scattered Data Approximation, Cambridge University Press, Cambridge, UK, 2005.

[49] M. W. WiLSON, Necessary and sufficient conditions for equidistant quadrature formula, SIAM J. Numer. Anal., 7 (1970), pp. 134-141.

Copyright (c) by SIAM. Unauthorized reproduction of this article is prohibited. 